\title{
Corrigendum to "Critical Incident Disclosing Behaviors and Associated Factors among Nurses Working in Amhara Region Referral Hospitals, Northwest Ethiopia: A Cross-Sectional Study"
}

\author{
Yeshiambew Eshete, ${ }^{1}$ Bekele Tesfaye, ${ }^{2}$ Zewdu Dagnew, ${ }^{2}$ Demewoz Kefale, ${ }^{1}$ \\ Demeke Mesfin Belay, ${ }^{1}$ and Binyam Minuye ${ }^{1}{ }^{1}$ \\ ${ }^{1}$ Department of Nursing, College of Health Sciences, Debretabor University, Debretabor, Ethiopia \\ ${ }^{2}$ College of Health Sciences, Debremarkos University, Debremarkos, Ethiopia \\ Correspondence should be addressed to Binyam Minuye; biniamminuye@yahoo.com \\ Received 11 April 2021; Accepted 11 April 2021; Published 3 May 2021
}

Copyright (c) 2021 Yeshiambew Eshete et al. This is an open access article distributed under the Creative Commons Attribution License, which permits unrestricted use, distribution, and reproduction in any medium, provided the original work is properly cited.

In the article titled "Critical Incident Disclosing Behaviors and Associated Factors among Nurses Working in Amhara Region Referral Hospitals, Northwest Ethiopia: A CrossSectional Study" [1], there was a spelling error in author Demeke Mesfin Belay's name in the author list, where "Demke Mesfin Belay" should have read "Demeke Mesfin Belay." This is corrected as shown in the author details above.

\section{References}

[1] Y. Eshete, B. Tesfaye, Z. Dagnew, D. Kefale, D. Mesfin Belay, and B. Minuye, "Critical Incident Disclosing Behaviors and Associated Factors among Nurses Working in Amhara Region Referral Hospitals, Northwest Ethiopia: A Cross-Sectional Study," Critical Care Research and Practice, vol. 2021, Article ID 8813368, 6 pages, 2021. 